\title{
THE INTERSTELLAR MEDIUM
}




\title{
THE X-RAY EMISSION OF E AND SO GALAXIES
}

\author{
G. FABBIANO \\ Harvard-Smithsonian CfA \\ 60 Garden St., Cambridge MA 02138, USA
}

\section{Introduction: Facts and Myths}

It is well known by now that $\mathrm{E}$ and $\mathrm{S} 0$ galaxies are associated with hot, $\mathrm{X}$ ray emitting gaseous halos, subject to central cooling flows, but otherwise homogeneous, which can be used to trace the galaxy potential and thus measure the total gravitational mass. Recent ASCA observations suggest that these halos have a surprisingly low metal abundance.

Although these 'facts' have entered the astronomical consciousness and do (or may) represent reality in some cases, if taken literally they may be more akin to myth. Although some galaxies are indeed associated with centrally cooling extensive X-ray halos, this is by no means true for all $\mathrm{E}$ and S0s. Although the X-ray data can be used to measure the binding mass of the galaxies, and in some cases the resulting $\mathrm{M} / \mathrm{L}$ are indeed large, these measurements cannot be applied blindly to all galaxies. Although ASCA spectra are returning the unmistakeable signature of thermally emitting plasma, how well do we understand these data and the physical state of the hot ISM?

In this paper, I attempt to review the observations critically and give my assessment on our present state of knowledge of the X-ray properties of E and S0 galaxies and on what we have learned about the structure and possibly evolution of these galaxies by using these data.

There is significant overlap between this paper and a talk I gave at the meeting on early-type galaxies in Mexico earlier this year (Fabbiano 1995).

\section{X-ray Bright and X-ray Faint E and So's}

Not all E and S0 galaxies retain their hot ISM. Some galaxies (e.g. NGC 4406 and NGC 4472 in Virgo; Forman et al 1979; Trinchieri, Fabbiano and Canizares 1986; Fabbiano, Kim and Trinchieri 1992) are associated with 
extensive halos clearly displaced from the stellar component, and in some cases extending to very large radii (e.g. NGC 4636; Trinchieri et al 1994). Other galaxies (Fabbiano, Gioia and Trinchieri 1989; Fabbiano, Kim and Trinchieri 1994) in similar environments, display much fainter X-ray emission, typically coextensive with the stellar body, and generally consistent with the emission expected from a population of X-ray sources similar to that of M31 (Fabbiano, Trinchieri and VanSpeybroeck 1987; Trinchieri and Fabbiano 1991).

The X-ray spectra of the X-ray bright galaxies show clearly the presence of line emission (e.g. Awaki et al 1994) thus confirming the presence of an optically thin hot ISM. The temperature of the emitting gas also appears to decrease at smaller radii (e.g. Trinchieri et al 1994; Kim and Fabbiano 1995), as it would be expected in the presence of cooling flows. X-ray faint galaxies have significantly different spectral distributions from those of Xray bright galaxies, suggesting the presence of multicomponent emission, with little - if any - hot ISM present (Fabbiano, Kim and Trinchieri 1994; Pellegrini and Fabbiano 1994).

The extent of the variation in the ability of retaining a hot gaseous halo by galaxies of a given optical luminosity is shown clearly when we plot the Einstein E and S0 sample in the $L_{X}-L_{B}$ plane. This diagram (Figure 1) shows an impressive amount of scatter. For a given $L_{B}$, one can find values of $L_{X}$ differing by two orders of magnitude. The lower $L_{X}$ galaxies have Xray emission in the range expected from a population of bulge-type stellar $\mathrm{X}$-ray sources.

\section{Correlation Studies}

We (Eskridge, Kim and Fabbiano $1995 \mathrm{a}, \mathrm{b}, \mathrm{c}$ ) explored the question of the important parameters affecting the presence of a hot ISM, and of the effect of the hot ISM on galaxy properties by studying the relations between stellar $\left(L_{12 \mu m}, L_{B}, L_{X}\right), I S M\left(L_{H I}, L_{100 \mu m}, L_{X}\right)$, nuclear $\left(L_{6 c m}\right)$, and structural [potential shape: $a / b, a_{4}$, , Bender at al 1989; potential depth: $\sigma_{v}, M g_{2}$, Faber 1973] parameters. We used censored analysis methods, including regression analysis and partial-rank tests.

We find that morphology is important, in the sense that S0s are systematically X-ray fainter than $\mathrm{E}$ galaxies, for a given optical luminosity. Although in this case the increased fraction of rotational energy in the former may play a role, it appears more likely that this effect is related to the shape of the potential (see also Pellegrini 1994; Ciotti and Pellegrini 1995).

There are stronger and more direct indications that the galaxy potential is a major player. The depth of the potential is a key factor in promoting the retention of a hot ISM. There are strong correlations between $L_{X}$ (and 


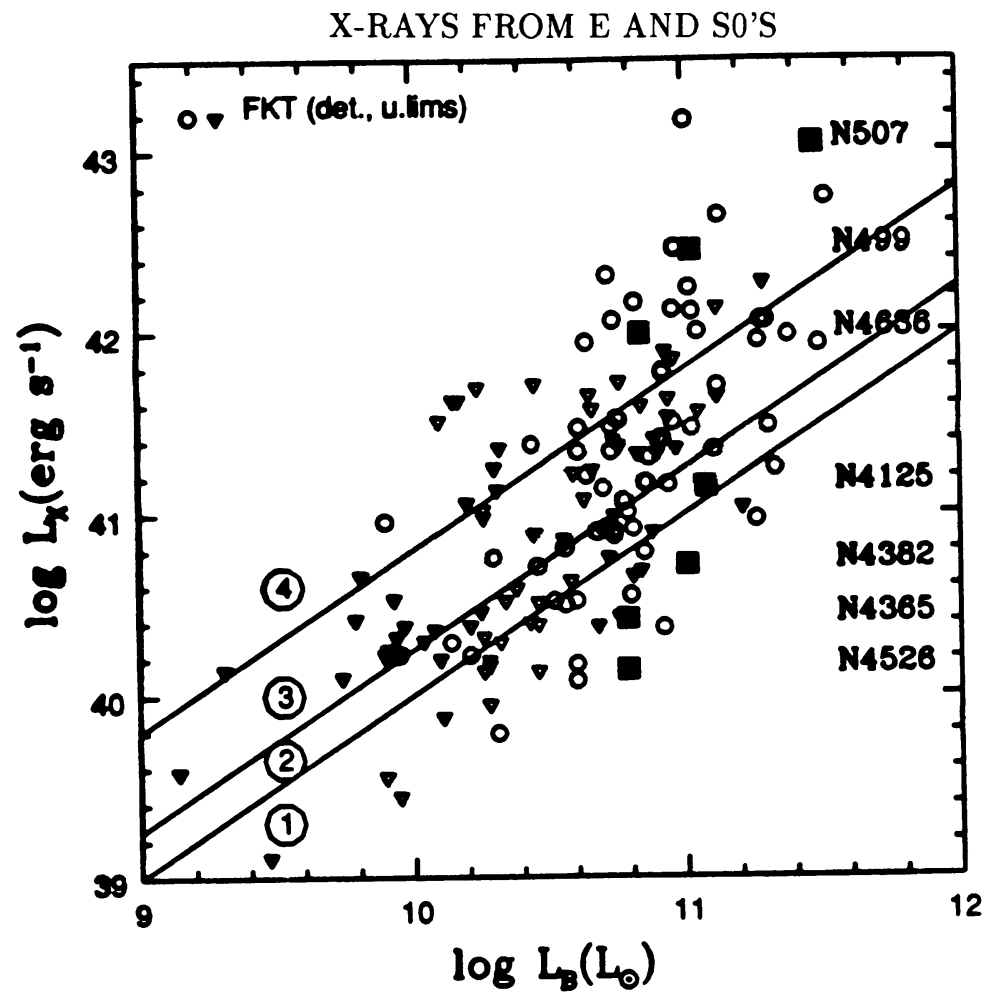

Figure 1. The distribution of the Einstein sample of $\mathrm{E}$ and $\mathrm{S} 0$ galaxies in the $L_{X}-L_{B}$ plane. The locus of Spirals and bulge dominated spirals overlaps regions 1 and 2 in this diagram.

the $L_{X} / L_{B}$ ratio) and both $\sigma_{v}$ and $M g_{2}$, suggesting that the deeper the potential well, the better the hot ISM retention, and the better the retention of stellar ejecta from the early formation phase. Also, a certain depth appears to be critical for both retaining the hot ISM and fuelling an active nucleus: there are 'threshold' correlations between $\sigma_{v}$ and both $L_{X} / L_{B}$ and $L_{6 \mathrm{~cm}}$.

The shape of the potential is also a key factor. Rounder (and boxier) galaxies have larger $L_{X}, L_{X} / L_{B}$, and $L_{6 c m}$, with the strongest correlation being that between $L_{X} / L_{B}$ and $a / b$. Also rounder galaxies tend to have boxier isophotes. These results persist when $\mathrm{E}$ and S0s are analyzed separately.

The potential shape and the depth parameters appear to be related, but not in a simple way. For instance, at a given value of $a_{4}$ the spread in $\sigma_{v}$ appears directly related to the spread in $M g_{2}$ such that the higher $\sigma_{v}$ objects also tend to have higher $M g_{2}$ (the $M g_{2}-\sigma_{v}$ relations). For a given $\sigma_{v}$ in the range where substantial X-ray halos are retained, boxy galaxies tend to have lower $M g_{2}$ values (and thus lower stellar abundances) than disky galaxies. However, the galaxies with the lowest $\sigma_{v}$ tend to have disky 
isophotes and low metallicity.

The coupling between the shape and depth parameters is clearly an important input for understanding the formation and evolution of early-type systems. A galaxy may have a deep potential either because of initial conditions, or because it is the product of mergers of a number of less massive objects (Bender et al 1992; Stiavelli 1992). In the case of relatively high $\sigma_{v}$ galaxies, for a given central velocity dispersion (depth of the potential), galaxies with disky inner isophotes tend to be more metal rich. If disky isophotes signal 'primordial' ellipticals, then a disky elliptical of a given mass would have higher abundance than a boxy elliptical, if the MassAbundance relationship is a relic of a fundamental contraint of the galaxy formation process. In this picture a boxy galaxy would be a merger product that was formed from lower mass (and thus lower abundance) precursors. Alternatively, disky isophotes may signal a merger system that has either formed a secondary nuclear disk (Hernquist and Barnes 1991), or captured a self-gravitating spiral disk. If so, then the higher $M g_{2}$ at a given $\sigma_{v}$ may be due to high abundance in those disks (Bender and Surma 1992).

The effect of merging is not clear. Merging was related to boxy isophotes and it was thus suggested that it may be a key factor for the presence of a hot ISM (Bender et al 1992). However, observations of galaxies believed to be remnants of recent mergers and found to be X-ray faint suggests that very recent merging may instead remove the hot ISM (e.g., NGC 4365, Fabbiano, Kim and Trinchieri 1994; NGC 3610 and NGC 4125, Fabbiano and Schweizer 1995). Perhaps the age of the merging event is a factor.

The link between $L_{X}$ and potential depth is confirmed by the comparisons with the $\kappa$ parameters of Bender, Burstein and Faber (1992), suggesting that $L_{X}$ is more strongly correlated with inner mass to light ratio than any other luminosity indicators. This suggests that galaxies with central excesses of dark matter also have more massive extended dark matter halos, providing a mechanism for retaining larger amounts of hot ISM. We also find that galaxies with higher inner $M / L$ for their mass also tend to be more metal rich. This may be due to the enhanced ability of such galaxies to mantain ongoing or episodic central star formation for extended periods, or to retain the enriched ejecta of early epochs of star formation, or both. This trend becomes stronger when tested for constant $a_{4}$, suggesting that this effect is not connected to the presence of metal-rich nuclear disks.

\section{Measuring the Binding Mass}

If $\mathrm{E}$ and $\mathrm{S} 0$ galaxies have hot gaseous halos in hydrostatic equilibrium in the galaxy potential, the X-ray data can be used to measure the mass of the galaxies (e.g. Fabricant and Gorenstein 1983). The use of the Einstein 
X-ray observation for measuring the gravitational mass of $\mathrm{E}$ and S0 galaxies (Forman, Jones and Tucker 1985) has been controversial, mostly because of the quality of the available data (Trinchieri, Fabbiano and Canizares 1986; see review in Fabbiano 1989).

Where are we ten years later? It is clear that any attempt at applying the standard hydrostatic equilibrium approach to observations of X-ray faint $\mathrm{E}$ and $\mathrm{S} 0$ is meaningless and will result in erroneous results, because the X-ray emission of these galaxies does not appear to be dominated by a hot gaseous halo (see above).

$\mathrm{X}$-ray bright $\mathrm{E}$ and $\mathrm{S} 0$ s retain hot gaseous halos, which indeed dominate the X-ray emission in the ROSAT range. With the ROSAT PSPC one can measure the radial temperature profile of these halos with reasonable accuracy (e.g. Trinchieri et al 1994; Trinchieri et al 1995, in preparation), a key measurement for contraining the binding mass (see Fabbiano 1989). These results point to $M / L \sim 20-50$ and thus to a considerable amount of dark matter in these galaxies. One must however remember that these mesurements are still dependent on assumptions on the spectral properties of the hot ISM, which in some cases are not unique (e.g. in NGC 4636, see Trinchieri et al 1994). One also assumes hydrostatic equilibrium.

A different approach was followed by Buote and Canizares (1994), who compared X-ray and optical isophotes of the flattened elliptical galaxy NGC 720, using ROSAT data. They found that the flattening of the Xray contours at large radii can only be explained if the galaxy contains an extended halo of dark matter.

Central group galaxies tend to have huge hot gaseous halos extending hundreds of kiloparsecs and $M / L \sim 100$ or more (e.g David et al 1994; Kim and Fabbiano 1995). These huge halos contain a sizeable fraction of the total mass of the system. Interestingly, these results may conflict with the ratio of baryonic to total mass expected in a $\Omega=1$ universe (see also Briel, Henry and Boehringer 1992). However, even here a note of caution is necessary, since clumpiness of the X-ray halo which may be undetected with the present instruments may lower the estimate of the baryonic mass. The ROSAT PSPC image of NGC 507 suggests indeed the presence of such clumps (Kim and Fabbiano 1995).

\section{Hot Halos and Active Nuclei}

A strong connection was found between the presence of a hot ISM and that of radio sources in $\mathrm{E}$ and $\mathrm{S} 0$ galaxies. In a statistical sense, there are clear strong correlations between $L_{X}, L_{X} / L_{B}$ and both total and nuclear radio power. These suggest that the hot ISM, accreting to the nuclei through cooling flows may be the fuel of the active nucleus. Moreover, the thermal 
pressure of this medium in X-ray bright galaxies is enough to represent an effective mechanism for confining and/or disrupting radio jets, and confining radio lobes in the less powerful radio galaxies (Fabbiano, Gioia and Trinchieri 1989).

We now begin to have direct evidence of the interaction between hot ISM and nuclear radio sources. The galaxy NGC 1399 is associated with a double lobed radio source, which is entirely contained in an extensive X-ray halo undergoing a cooling flow (Killeen and Bicknell 1988). We have obtained a high resolution ROSAT HRI image of the central regions of this galaxy (Kim et al 1995). This image shows a remarkable inhomogeneity of the ISM in a variety of scales, including an arcmin scale spiral-arm like feature; an E-W flattened central region with a total extent of about 1' and cooler than the surrounding regions; a few unresolved clumps; and finally a remarkable tunnel-like structure enveloping the northern radio lobe, with a possible suggestion of a similar structure in the south as well (see figure in Fabbiano 1995). The thermal pressure we derive from the tunnel's walls is in excess of that in the radio lobes. Thermal confinement is therefore the likely mechanism in this galaxy. Interactions between radio jets and hot ISM are also suggested in NGC 1316 (Kim, Fabbiano and Mackie in preparation).

These X-ray images suggest complexity in the hot ISM of early-type galaxies, and give us a taste of what is to come when AXAF will be returning high spectral and spatial resolution data on these galaxies.

\section{Different Phases of the ISM}

The relations between different phases of the ISM of early-type galaxies (hot - X-ray; warm - optical emission line; cold - FIR, HI), have been the subject of a number of papers (e.g. Trinchieri and diSerego Alighieri 1991; Bregman, Hogg and Roberts 1992; Eskridge, Fabbiano and Kim 1995a). These works suggest, but do not prove, a possible connection between the presence of cooling flows and $\mathrm{H} \alpha$ emission. They also show that the presence of a hot ISM and of cold HI gas or dust (FIR) are largely uncorrelated, suggesting either different origins (in the case of the HI), or an adverse effect of the hot X-ray emitting environment (in the case of dust).

Detailed comparisons of X-ray images of galaxies and images at other wavelengths are now being pursued and are beginning to reveal a rich multiphase ISM. In NGC 1316, dust lanes and hot ISM coexist in the core, as well as $\mathrm{H} \alpha$ emitting gas (Kim et al, in preparation). 


\section{The Abundance Question}

The analysis of the ASCA CCD observations of galaxies provide far greater resolution than available with proportional counter data, leading to the detection of line emission. Using these data, significant subsolar metal abundance have been reported in the hot ISM of E and S0 galaxies (Awaki et al 1994; Matsushita et al 1994). These reports are in definite contrast with the expectations from stellar measurements and theory, which would predict metallicities of at least solar in the ISM (see N. Arimoto's talk, this volume).

A discussion of the possible pitfalls of the present X-ray spectral analysis, that may lead to spurious results as far as the abundances are concerned, can be found in Fabbiano (1995). Here I will not repeat those argument, but just state that this question is generating a lively amount of debate in the X-ray community. The low-abundance problem is not limited to elliptical galaxies. Similar puzzling results have been reported from observations of stars, for which abundances are well known (e.g. AR Lac, observed with ASCA, White et al 1994; and other stars, observed with both ROSAT and ASCA, J. Bregman 1995, private communication). Also the ASCA observation of the Cygnus Loop has been reported to suggest subsolar abundances (Miyata et al 1994). Unless we want to accept that metals are depleted in the X-ray emitting regions in these systems (interestingly, the opposite -if anything- may happen in the Sun; Meyer 1991, Phillips et al 1994), we have to conclude that there is something wrong with either our choice of models or our fitting procedures.

I think that before subscribing to the low-abundance interpretation of the spectral results on elliptical galaxies, we need to understand better what is going on with other less controversial astrophysical plasmas.

This work was partially supported by NASA contract NAS8-39073 (AXAF Science Center), and by NASA grant NAGW2681 (LTSA).

\section{References}

Awaki, H. et al 1994, P. A. S. J., 46, L65.

Bender, R., Surma, P., Doebereiner, S., Moellenhoft, C., and Madejsky, R. 1989, Astr. Ap., 217, 35.

Bender, R. et al 1992, in "Structure, Dynamics, and Chemical Evolution of Elliptical Galaxies”, I. J. Danziger, W. W. Zeilinger, and K. Kjar, eds., ESO Proc. No. 45, p. 3.

Bender, R. and Surma, P. 1992, Astr. Ap., 258, 250.

Bender, R., Burstein, D., and Faber, S. M. 1992, Ap. J., 399, 462.

Briel, U. G., Henry, J. P., and Boehringer, H. 1992, Astr. Ap., 259, L31.

Buote, D., and Canizares, C. R. 1994, Ap. J., 427, 86.

Ciotti, L. and Pellegrini, S. 1995, M. N. R. A. S., submitted.

David, L. P., Jones, C., Forman, W., and Daines, S. 1994, Ap. J., 428, 544. 
Eskridge, P. B., Fabbiano, G., and Kim, D.-W. 1995a, Ap. J. Suppl., 97, 141.

Eskridge, P. B., Fabbiano, G., and Kim, D.-W. 1995b, Ap. J., 442, 523.

Eskridge, P. B., Fabbiano, G., and Kim, D.-W. 1995c, Ap. J., in press.

Fabbiano, G. 1989, Ann. Rev. Ast. Ap., 27, 87.

Fabbiano, G. 1995, in "Fresh Views on Elliptical Galaxies", A. Buzzoni, ed., Pub. of A. Soc. P., in press.

Fabbiano, G., Kim, D.-W., and Trinchieri, G. 1992, Ap. J. Suppl., 80, 531.

Fabbiano, G., Gioia, I. M., and Trinchieri, G. 1989, Ap. J., 347, 127.

Fabbiano, G., Kim, D.-W., and Trinchieri, G. 1994, Ap. J., 429, 94.

Fabbiano, G., and Schweizer, F. 1995, Ap. J., in press.

Fabbiano, G., Trinchieri, G., and VanSpeybroeck, L. 1987, Ap. J., 316, 127.

Faber, S. M. 1973, Ap. J., 179, 731.

Fabricant, D., and Gorenstein, P. 1983, Ap. J., 267, 535.

Forman, Jones, C. and Tucker, W., 1985, Ap. J., 293, 102.

Forman, W., Schwarz, J., Jones, C., Liller, W., and Fabian, A. C. 1979, Ap. J., 234, L27.

Hernquist, L., and Barnes, J. E. 1991, Nature, 354, 210.

Killeen, N.E.D. and Bicknell, G.V. 1988, Ap. J., 325, 169.

Kim, D.-W., and Fabbiano, G. 1995, Ap. J., 441, 182.

Kim, D.-W., Fabbiano, G., Mackie, G., and Norman, C. 1995, Ap. J., submitted.

Matsushita, K. et al 1994, Ap. J., 436, L41.

Meyer, J. 1991, Adv. Space Res., 11, 269.

Miyata, E., Tsunemi, H., Pisarski, R., and Kissel, S. E. 1994, P. A. S. J., 46, L101.

Pellegrini, S. 1994, Astr. Ap., 292, 395.

Pellegrini, S. and Fabbiano, G. 1994, Ap. J., 429, 105.

Phillips, K. J. H., Pike, C. D., Lang, J., Watanabe, T., and Zarro, D. M. 1994, Proc. of Kofu Symp., NRO Report No. 360, p. 301.

Stiavelli, M. 1992 in "Structure, Dynamics, and Chemical Evolution of Elliptical Galaxies", I. J. Danziger, W. W. Zeilinger, and K. Kjar, eds., ESO Proc. No. 45, p. 303.

Trinchieri, G., and di Serego Alighieri, S. 1991, A. J., 101, 1647.

Trinchieri, G., and Fabbiano, G. 1991, Ap. J., 382, 82.

Trinchieri, G., Fabbiano, G., and Canizares, C. R. 1986, Ap. J., 310, 637.

Trinchieri, G, Kim, D.-W., Fabbiano, G., and Canizares, C. R., 1994, Ap. J., 428, 555.

White, N. E. et al 1994, P. A. S. J., 46, L97.

Inge Thiering: Concerning the metallicity problem you reviewed, I would like to comment that for our ROSAT PSPC observations of the groups around the first ranking galaxies NGC 4104, NGC 6269, and NGC 6329 we obtain spectral fits with $\chi^{2} / \nu \sim 1$ for two different models of the hot gas: one is assuming a two-component gas of variable temperature and $z / z_{0} \sim 1$, and the other assumes a roughly isothermal gas whose metallicity decreases from the inside to the ouside from $z / z_{\circ} \sim 1$ to $z / z_{o} \sim 0.1-0.3$.

Answer: Thanks for your comment. I agree that with the PSPC one cannot find a unique model (see also Trinchieri et al 1994; Fabbiano, Kim and Trinchieri 1994).

Duncan Forbes: Do you see a morphological coincidence between soft X-rays $\left(10^{6} \mathrm{~K}\right)$ and $\mathrm{H} \alpha$ emission $\left(10^{4} \mathrm{~K}\right)$ ?

Answer: In general, $\mathrm{H} \alpha$ is found in the cores of galaxies, where cooling may occur. However, I am not aware of detailed one to one spatial comparisons for a large enough sample of galaxies. 